\title{
Risk of prevalent and incident dementia associated with insulin-like growth factor and insulin-like growth factor binding-protein 3
}

Osvaldo P. Almeida, $\mathrm{PhD}^{1,2,3}$

Graeme J. Hankey, $\mathrm{MD}^{1,4}$

Bu B. Yeap, $\mathrm{PhD}^{1,5}$

S.A. Paul Chubb ${ }^{1,5}$

Jonathan Golledge, MChir ${ }^{7,8}$

Leon Flicker, $\mathrm{PhD}^{1,2,9}$

${ }^{1}$ Medical School, University of Western Australia, Perth, Australia

${ }^{2}$ WA Centre for Health \& Ageing of Centre for Medical Research, Harry Perkins Institute of Medical Research, Perth, Australia

3 Department of Psychiatry, Royal Perth Hospital and Bentley Hospital, Perth, Australia

${ }^{4}$ Department of Neurology, Sir Charles Gairdner Hospital, Perth, Australia

${ }^{5}$ Department of Endocrinology, Fiona Stanley Hospital, Perth, Australia

${ }^{7}$ Queensland Research Centre for Peripheral Vascular Disease, College of Medicine and Dentistry, James Cook University, Townsville, Australia

${ }^{8}$ Department of Vascular and Endovascular Surgery, The Townsville Hospital, Townsville, Australia

${ }^{9}$ Department of Geriatric Medicine, Royal Perth Hospital, Perth, Australia

Running title: Dementia, IGF-1 and IGFBP-3

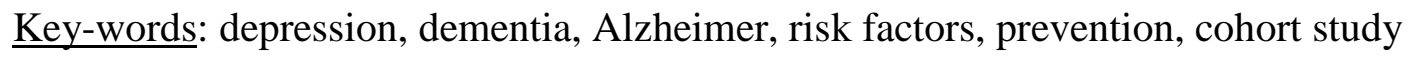

Correspondence

Prof. Osvaldo P. Almeida, School of Psychiatry \& Clinical Neurosciences (M573), University of Western Australia, 35 Stirling Highway, Crawley, Perth, WA 6009, Australia. Email: osvaldo.almeida@uwa.edu.au. 


\section{ABSTRACT}

Insulin-like growth factor 1 (IGF-1) influences cell proliferation and survival. In the extracellular environment, IGF-1 circulates bound to proteins (IGFBP), some of which have physiological effects that seem independent of IGF-1, including the brain (e.g., IGFBP-3). We completed a systematic review of the association between dementia and IGF-1 and IGFBP-3, and a crosssectional and longitudinal study designed to investigate if lower plasma concentration of these proteins increased the risk of prevalent and incident dementia. 3967 men aged 71 to 89 years joined the study, of whom 535 (13.5\%) showed evidence of prevalent cognitive impairment. The plasma concentrations of IGF-1 and IGFBP-3 were similar for men with and without cognitive impairment. The 3432 men free of cognitive impairment were then followed for up to 13 years. During this time 571 (16.6\%) developed dementia. The plasma concentration of IGF-1 had no association with incident dementia. The doubling of the plasma concentration of IGFBP-3 decreased the hazard ratio of dementia by $23 \%$ (95\%CI=5\% to $37 \%)$. The results were not affected by age, body mass index and history of smoking, diabetes, hypertension, coronary heart disease or stroke. If these findings are confirmed by others, the plasma concentration of IGFBP-3 could be used to improve the accuracy of predictive models of dementia and as a potential new factor to assist in the development of prevention and treatment strategies. 


\section{INTRODUCTION}

Insulin-like growth factor 1 (IGF-1) is a hormone that is structurally similar to the pro-insulin peptide and mediates, among other things, the growth-promoting effects of the human growth hormone. ${ }^{1}$ Most IGF-1 circulates bound to other proteins, which are known as IGF binding proteins (IGFBP). Six IGFBP have been described to date (IGFBP 1 to 6 - see reference for detailed review of their physiological functions), ${ }^{2}$ with IGFBP-3 recently attracting attention because of its various physiological effects that seem largely independent of IGF-1. ${ }^{3}$ For example, currently available evidence suggests that IGFBP-3 could contribute to mediate cell proliferation, death and survival. ${ }^{3}$ In later life, lower concentration of IGFBP-3 has been associated with higher mortality rates, ${ }^{4}$ while IGFBP-3 overexpression has been observed in some cancers ${ }^{2}$ and in the brains of people who have died with Alzheimer's disease (AD). ${ }^{5}$ Given these findings, it is not surprising that several groups have investigated the association between dementia and IGF-1 and IGFBP-3.

Seven cross-sectional studies have examined the relationship between dementia and IGF-1. ${ }^{6}$ Taken together, their results suggest that people with dementia $(n=616)$ have lower circulating IGF-1 than healthy controls ( $n=726)$. However, there was evidence of marked study heterogeneity, sub-optimal methodological quality and publication bias (supplementary material, eTables 1 and 2 and eFigure 1). Similar methodological problems beset the 6 studies that compared IGFBP-3 in people with $(n=510)$ and without dementia (n=493) (supplementary material, eTables 1 and 2 and eFigure 1). Hence, existing evidence suggests that the nature of the association between dementia and IGF-1 and IGFBP-3 remains uncertain.

There are limited data investigating the longitudinal association between IGF-1 or IGFBP-3 and incident dementia. The Caerphilly study used blood samples from 745/2512 (29.7\%) men who subsequently underwent cognitive assessment over a 20-year period. ${ }^{7}$ They found no obvious association between baseline concentration of IGF-1 or IGFBP-3 and incident dementia or 
cognitive impairment, ${ }^{7}$ although selective sampling and differential loss to follow up created uncertainty about the validity and generalizability of the findings.

The Health In Men Study (HIMS) has been collecting longitudinal data from a communityrepresentative sample of older men since 1996. During the 2001-2004 assessment, about 4000 participants donated a blood sample and have since been followed continually using the Western Australian Data Linkage System (WADLS), ${ }^{8}$ thereby circumventing concerns about differential loss to follow up. We used HIMS to investigate the cross-sectional and longitudinal association between the plasma concentration of IGF-1 and IGFBP-3 and cognitive impairment or dementia. Based on our systematic review of the topic (supplementary material), we hypothesised that participants with dementia would have lower plasma concentration of both IGF-1 and IGFBP-3, and that lower baseline concentration of IGF-1 and IGFBP-3 would be associated with greater risk of incident dementia over 10 years than high concentrations. ${ }^{4}$

\section{METHODS}

Study design, setting and participants

HIMS is a cohort study of a community-representative sample of older men living in the Perth metropolitan region of Western Australia, Australia. Details about the recruitment of participants have been described elsewhere. ${ }^{9}$ Briefly, 4243 men donated a blood sample during the 2001-2004 wave of assessments and valid assays for IGF-1 and IGFBP-3 were available for 3967 participants (93.5\%). The flow of participants in this study is summarised in eFigure 2. Their age ranged from 71 to 89 years and together they formed the sample for this investigation. They were followed until they received the diagnosis of dementia, died or the $30^{\text {th }}$ June 2015, whichever occurred first. Participants provided written informed consent and all study procedures were approved by the Human Research Ethics Committee of the University of Western Australia. 


\section{Outcomes: cognitive impairment and dementia}

During the 2001-2004 assessment we examined participants with the Mini-Mental State Examination (MMSE) and considered that those with a total score of 23 or less had cognitive impairment. ${ }^{10}$ In addition, we retrieved data from WADLS to determine if a diagnosis of dementia had been recorded between $1^{\text {st }}$ January 1980 and $30^{\text {th }}$ June 2015. WADLS incorporates information for all health contacts in Western Australia, including emergency departments, elective and nonelective admissions, mental health outpatient services, community aged care services, as well as cancer and death registries. ${ }^{8}$ Each occasion of service leads to the recording of the date and the relevant primary and up to 20 secondary diagnoses that are coded according to the International Classification of Diseases (ICD). The following ICD-9 and ICD-10 codes indicate the presence of dementia: 290, 294.1, 294.2, 331.0, 331.1, 331.2, 331.82, and F00, F01, F02, F03, G30, G31.0, G31.1 and G31.83. We made no attempt to examine individual types of dementia because 'unspecified dementia' was the most frequently recorded diagnosis. The diagnoses recorded in WADLS rely on all clinical data available at the time, including information obtained from the next-of-kin, general practitioners, medical notes and investigations. WADLS does not record the sources of information used to reach a diagnosis, only that a diagnosis was established.

In this study, we considered that prevalent cognitive impairment was present if the MMSE score was $\leq 23$ or if a diagnosis of dementia had been recorded in WADLS prior to the 2001-2004 assessment. For the longitudinal study, we excluded from the sample men with prevalent cognitive impairment (as described above) and used WADLS to establish the onset of dementia during follow up. For the longitudinal study, the baseline date was the date of the assessment of each participant during 2001-2004 wave.

Exposures: IGF-1 and IGFBP-3 
We collected blood samples from participants between 08:00 and 10:30 AM during the 2001-2004 visit. Plasma was separated immediately after collection and stored at -80C until assayed. We used commercial reagent kits of single lot numbers to measure IGF-1 and IGFBP-3 (Diagnostics Systems Laboratories - supplied by Beckman Coulter, Gladesville, NSW, Australia). A Grifols Triturus ELISA processor was used to automate the assays (Vital Diagnostics, Castle Hill, NSW, Australia). Coefficients of variation were $12.2 \%$ and $8.6 \%$ for IGF-1 concentrations of 117 and $216 \mathrm{ng} / \mathrm{mL}$ respectively, and $16.8 \%$ and $4.4 \%$ for IGFBP-3 concentrations of 540 and $4300 \mathrm{ng} / \mathrm{mL}$ respectively.

\section{Other study measures}

At the 2001-2004 assessment participants provided information about their age (in years), date of birth, educational achievement (completed high school vs incomplete high school - recorded at the time of recruitment), and smoking (never, past or current). We used a self-report questionnaire to establish the presence of diabetes, hypertension, coronary heart disease and stroke: "Have you ever been told that you have diabetes (yes/no), hypertension (yes/no), angina (yes/no), heart attack (yes/no), heart bypass surgery (yes/no), heart balloon angioplasty (yes/no), stroke (yes/no)”. We considered that men had coronary heart disease if they answered "yes” any of the questions about angina, heart attack, heart bypass surgery or heart balloon angioplasty. We used a standardised scale to measure the weight (in kilograms - light clothing, no shoes) and the height (in centimetres) of participants, and calculated their body mass index (BMI) using the formula: BMI=weight/(height/100) $)^{2}$.

This survey did not have access to information about history of dementia in the family of participants.

\section{Statistical analyses}


We managed and analysed the data with the statistical package Stata 14.2 (StataCorp, College Station, Texas 77845, USA). We used descriptive statistics to summarise study measures as counts, proportions, range, mean and standard deviation of the mean (SD), and compared men with and without cognitive impairment at study entry using Pearson's chi-square statistic $\left(X^{2}\right)$ and t-tests. In the case of IGF-1 and IGFBP-3 concentrations, the analyses were further adjusted for the confounding effect of age, educational attainment, and history of coronary heart disease and stroke using analysis of variance.

We subsequently excluded from the analyses men with prevalent cognitive impairment and calculated the risk of incident dementia using a range of approaches. First, we calculated the doubling of IGF-1 and IGFBP-3 by dividing the natural log of these measures by the natural log of 2, and then used Cox regression to estimate the crude and adjusted hazard ratio (HR) of dementia (analyses adjusted for age and history of smoking, diabetes and stroke) resulting from the doubling of IGF-1 and IGFBP-3. Second, we grouped participants into quintiles of IGF-1 and IGFBP-3, and estimated the hazard ratio of dementia for each quintile using the lowest quintile as the reference. Again, the analyses were later adjusted for age and history of smoking, diabetes and stroke. Finally, we calculated the probability of dementia according to the plasma concentration of IGF-1 and IGFBP-3 using the "predict" subcommand of Stata following a logistic regression analysis adjusted for age and history of smoking, diabetes and stroke - the findings were plotted in graphic form using the "twoway qfitci” command of Stata.

We also completed a series of post-hoc analyses designed to ensure that the possible association between dementia and IGF-1 and IGFBP-3 concentrations was not due to differential mortality or to the inclusion of false negative cases of dementia in the cohort. We used competing risk regression to address the former (risk ratio expressed as sub-hazard ratio - SHR and the respective 95\%CI) and dealt with the latter by repeating the analyses after excluding men who had developed dementia 
or had died during the initial 2 years of follow up. The codes used for the statistical analyses are available from the corresponding author.

The results of all analyses reported in this manuscript were two-tailed and alpha was set at 5\%.

\section{Sample size}

We estimated that the study would require a sample size of 3153 participants to declare as significant a hazard ratio of 0.8 associated with an event probability of $20 \%$ (power $80 \%$ ). The study had access to 3432 men for the longitudinal study.

\section{RESULTS}

The mean \pm SD age of the 3967 participants was $77.0 \pm 3.6$ years. Of these, 535 (13.5\%) showed evidence of cognitive impairment (only 15 men with diagnosis of dementia in WADLS did not have MMSE $\leq 23$ ). Table 1 summarises the sociodemographic, lifestyle and clinical characteristics of men with and without cognitive impairment. Men with cognitive impairment were, on average, nearly one year older $(77.8 \pm 3.8$ vs $76.9 \pm 3.6$ years; $\mathrm{t}=5.21, \mathrm{p}<0.001)$. In addition, a lower proportion of them had completed high school, and the prevalence of coronary heart disease and stroke was higher amongst those with cognitive impairment. The plasma concentration of IGF-1 was similar among men with and without cognitive impairment. The plasma concentration of IGFBP-3 was lower among men with than without cognitive impairment, but the difference between the groups disappeared once we took into account in the analysis the effects of age, education and history of coronary heart disease and stroke (Table 1).

\section{TABLE 1}


We then followed the 3432 men without cognitive impairment for $9.2 \pm 3.4$ years (range: 0.1 to 13.6 years). During this time, 571 (16.6\%) developed dementia and 1230 (35.8\%) died free of dementia. The doubling in the plasma concentration of IGF-1 at study entry was associated with a HR of dementia of 1.03 (95\%CI=0.90 to 1.18 ; $\mathrm{HR}=1.04,95 \% \mathrm{CI}=0.91$ to 1.18 after adjustment for age, smoking group, and history of diabetes, coronary heart disease and stroke, and body mass index at baseline). In contrast, doubling of the plasma concentration of IGFBP-3 decreased the HR of dementia during follow up $(\mathrm{HR}=0.73,95 \% \mathrm{CI}=0.59$ to $0.89 ; \mathrm{HR}=0.77,95 \% \mathrm{CI}=0.63$ to 0.95 after adjustment for age, smoking group, and history of diabetes, coronary heart disease, stroke, and body mass index at baseline). Men in the lowest quintile of IGFBP-3 concentration had 47\% (95\%CI=1\% to $64 \%$ ) greater risk of developing dementia than their counterparts in the highest quintile of IGFBP-3 concentration $(41 \%, 95 \% \mathrm{CI}=8 \%$ to $84 \%$ after adjustment for age, smoking group, and history of diabetes, coronary heart disease and stroke, and body mass index at baseline). We did not attempt to use multiple imputation for these multivariate analyses because there were missing data for 10 participants only.

We subsequently grouped participants into quintiles of IGF-1 and IGFBP-3 concentrations and reran the Cox regression analyses. The results of these analyses are summarised in Table 2. The plasma concentrations of IGFBP-3 for each quintile were, in order: 603 to $3010 \mathrm{ng} / \mathrm{mL}, 3020$ to $3560 \mathrm{ng} / \mathrm{mL}, 3570$ to $4020 \mathrm{ng} / \mathrm{mL}, 4030$ to $4530 \mathrm{ng} / \mathrm{mL}$ and 4540 to $7600 \mathrm{ng} / \mathrm{mL}$. Figure 1 shows the adjusted probability of incident dementia according to the plasma concentration of IGF-1 and IGFBP-3.

\section{FIGURE 1}

Post-hoc analyses 
We re-ran our analyses using death as a competing risk for dementia. The doubling of IGFBP-3 was associated with a SHR of dementia of 0.72 (95\%CI=0.59 to 0.88 - analyses adjusted for age, smoking group, and history of diabetes, coronary heart disease and stroke, and body mass index at baseline). Similarly, the SHR of dementia associated with IGFBP-3 quintiles was, in order, 0.84 (95\%CI=0.66 to 1.07$), 0.89(95 \% \mathrm{CI}=0.70$ to 1.13$), 0.68 \quad(95 \% \mathrm{CI}=0.52$ to 0.88$)$ and 0.69 (95\%CI $=0.53$ to 0.90 ). Twenty-three men developed dementia and 127 died during the initial 2 years of follow up. After excluding these participants from the analyses, we found that the adjusted SHR of dementia associated with the doubling of IGFBP-3 remained largely unchanged at 0.72 (95\%CI=0.59 to 0.89 ). The respective adjusted SHR of dementia associated with IGFBP-3 quintiles for this subsample was 0.88 ( $95 \% \mathrm{CI}=0.69$ to 1.13$), 0.90$ (95\%CI=0.70 to 1.15$), 0.68$ (95\%CI=0.52 to 0.89 ) and 0.70 (95\% CI=0.54 to 0.92$)$. These associations did not change after we excluded from the analyses participants who had developed dementia or had died within the first 4 years of follow up - this happened despite progressive loss of power (data not shown). Finally, we re-ran our analyses after excluding participants with the lowest and highest $1 \%$ of values if IGFBP-3. The crude HR of dementia associated with the doubling of IGFBP-3 concentration was 0.65 (95\%CI $=0.51$ to 0.83$)$ and the adjusted HR 0.69 (95\%CI=0.54 to 0.88 - adjusted for age, smoking group, and history of diabetes, coronary heart disease and stroke, and body mass index at baseline).

\section{DISCUSSION}

The results of this study showed that the plasma concentration of IGF-1 is not associated with cognitive impairment either cross-sectionally or longitudinally. In contrast, we found that the concentration of IGFBP-3 is lower among older men with cognitive impairment (although the association was no longer significant after adjustment for other study measures) and is inversely associated with incident dementia. Doubling of the plasma concentration of IGFBP-3 decreased the risk of incident dementia by about 25\%. Men in the lowest quintile of IGFBP-3 concentration had, 
on average, $40 \%$ greater risk of developing dementia over a 9-year period than their counterparts in the highest quintile of concentrations.

This project has the merit of having used a community-derived sample of older men recruited through random sampling of the Western Australian Electoral Roll (voting in Australia is mandatory for all adults aged 18 years or over), ${ }^{9}$ although those who were frail were less likely to be available for assessment. ${ }^{11}$ Hence, our results apply to a group of older men living in the community who were free of significant functional impairments at the time of enrolment - this would have excluded people with moderate to severe dementia from our cross-sectional analyses, which would have biased the results towards the null hypothesis. Such bias would not have affected the longitudinal study (people with prevalent cognitive impairment were excluded), and this may explain why the inverse association between IGFBP-3 and dementia risk could only be confirmed for incident cases. We are also mindful of the fact that we recruited a sample of men aged 70 years or over, and that the associations that we observed may not necessarily apply to women or younger men. For example, hypertension in mid, but not in later life, is strongly associated with the development of dementia. ${ }^{12}$ It is conceivable that the risk of dementia associated with exposure to IGF-1 and IGFBP-3 in early and late life may differ, but a prolonged longitudinal study starting in midlife would be required to clarify such a possibility. Finally, we acknowledge we were unable to contrast the predictive value of IGFBP-3 concentration with well-established risk factors for dementia, such as the ApoE4. We are not aware of any data suggesting that ApoE4 could mediate some of the effects of IGFBP-3, but this is an issue that will require the attention of future studies.

We used the MMSE and WADLS to establish the presence of cognitive impairment in our crosssectional study. The MMSE is a valid and widely used measure to establish the presence of cognitive impairment, ${ }^{10}$ which we enriched by using WADLS (2.8\% of the men with prevalent cognitive impairment had MMSE $\geq 24$ ). The longitudinal arm of the study benefited from 
negligible loss to follow up, as all health contacts and deaths are recorded by WADLS and the migratory movement of older Western Australians is minimal. ${ }^{13}$ We are also confident that our findings on incident dementia were not due by contamination of the sample with prevalent cases, as the results remained unchanged in our sensitivity analyses. We concede, however, that the diagnosis of dementia retrieved from WADLS was not based on a structured clinical assessment, but on information retrieved from hospital and aged care community services. This creates some uncertainty regarding the accuracy of the diagnosis of dementia in the study. Existing evidence indicates that WADLS has good specificity for the diagnosis of dementia (negligible number of false positive cases), but its sensitivity (particularly for mild cases) may be suboptimal. ${ }^{14}$ The longitudinal design of the study may have contributed to mitigate the impact of such a bias on the calculation of incidence rates (as cases are eventually recorded over time as the disease progresses), and, indeed, the incidence rates that we observed are consistent with those reported by others using different methodologies. ${ }^{15}$ This suggests that the approach we used to calculate risk estimates is likely to have produced robust results.

We should also consider the possibility of misclassification and bias associated with low IGFBP-3. Lower plasma concentration of IGFBP-3 has been associated with increased morbidity and mortality. ${ }^{2,3}$ Thus, people with low concentration of IGFBP-3 may more promptly and frequently come to medical attention, which would then increase the opportunity for the diagnosis of dementia to be made earlier. This could potentially bias this association, in which case the real risk of dementia associated with low IGFBP-3 could be less robust than our data suggest. Finally, we acknowledge that residual confounding and random error could conceivably explain some of our findings, as could confounding due to unmeasured factors (e.g., nutritional status, alcohol use, IGF2). For example, IGF-1 and IGF-2, together with the acid-labile subunit, form stable complexes with circulating IGFBP- $3,^{3}$ so that low concentration of IGFBP-3 may imply low concentration of 
IGF-2 when the concentration of IGF-1 remains unchanged. As information on IGF-2 was not available for this study, we could not examine its effect on cognitive outcomes.

Four of the seven cross-sectional studies currently available found that people with dementia have lower plasma concentration of IGFBP-3 than healthy controls, ${ }^{16-18} 2$ found no difference between the groups, ${ }^{19,20}$ and 1 reported higher IGFBP-3 concentrations among older adults with dementia. ${ }^{21}$ One longitudinal survey found no obvious association between IGFBP-3 and risk of dementia, although follow up cognitive data were only available for less than half of the original sample (our study had follow up data for all participants). ${ }^{7}$ A recent investigation designed to clarify the role of IGFBP-3 in the brain found that IGFBP-3 null mice (mice engineered to not express IGFBP-3) display deficiencies in neuronal structure and signalling. ${ }^{22}$ These mice also showed behavioural abnormalities suggestive of impaired working memory and visuospatial difficulties. Such findings indicate that IGFBP-3 contributes to modulate certain aspects of brain function and that IGFBP-3 deficient brains may be more vulnerable to cognitive deficits. There is also some evidence that IGFBP-3 contributes to repair damaged DNA, but inhibits cell replication and promotes cell death when there is marked genomic damage. ${ }^{3}$ If the results of basic research could be translated to humans, one might speculate that the brains of people with low circulating IGFBP-3 may be less efficient at repairing genomic damage and that this would lead to an increase in the rate of brain cell loss and, ultimately, dementia. Future studies should seek to replicate our findings and clarify whether the association between IGFBP-3 and dementia is truly causal. Should that be the case, the plasma concentration of IGFBP-3 may prove to be a useful marker of dementia risk and, potentially, offer new insights into how to prevent and treat dementia.

In summary, we found that IGF-1 was not associated with either prevalent or incident dementia, whereas low plasma concentration of IGFBP-3 was associated with increased probability of incident dementia over a 9-year period. If these results are confirmed by others, the plasma 
concentration of IGFBP-3 could be used to improve the accuracy of predictive models and as a potential new factor that might assist in the prevention and treatment of dementia.

\section{ACKNOWLEDGEMENTS}

The authors thank research staff and study participants for their generous contribution.

\section{CONFLICT OF INTEREST}

This work was funded through competitive project grants from the National Health and Medical Research Council of Australia (NHMRC), numbers 279408, 379600, 403963, 513823, 540403, 540504, 540405, 634492, 1021416, 1045710 and 1060557. The sponsors had no role in the design and conduct of the study; collection, management, analysis, and interpretation of the data; or preparation, review, or approval of the manuscript. The authors declare they have no conflict of interest. 


\section{REFERENCES}

1. Clemmons DR. Clinical utility of measurements of insulin-like growth factor 1. Nat Clin Pract Endocrinol Metab 2006; 2: 436-446.

2. Baxter RC. IGF binding proteins in cancer: mechanistic and clinical insights. Nat Rev Cancer 2014; 14: 329-341.

3. Baxter RC. Insulin-like growth factor binding protein-3 (IGFBP-3): Novel ligands mediate unexpected functions. J Cell Commun Signal 2013; 7: 179-189.

4. Yeap BB, Chubb SA, McCaul KA, Ho KK, Hankey GJ, Norman PE et al. Associations of IGF1 and IGFBPs 1 and 3 with all-cause and cardiovascular mortality in older men: the Health In Men Study. Eur J Endocrinol 2011; 164: 715-723.

5. Watanabe K, Uemura K, Asada M, Maesako M, Akiyama H, Shimohama S et al. The participation of insulin-like growth factor-binding protein 3 released by astrocytes in the pathology of Alzheimer's disease. Mol Brain 2015; 8: 82.

6. Hu X, Yang Y, Gong D. Circulating insulin-like growth factor 1 and insulin-like growth factor binding protein-3 level in Alzheimer's disease: a meta-analysis. Neurol Sci 2016; 37: 16711677.

7. Green CJ, Holly JM, Bayer A, Fish M, Ebrahim S, Gallacher J et al. The role of IGF-I, IGF-II, and IGFBP-3 in male cognitive aging and dementia risk: the Caerphilly Prospective Study. $J$ Alzheimers Dis 2014; 41: 867-875.

8. Holman CD, Bass AJ, Rosman DL, Smith MB, Semmens JB, Glasson EJ et al. A decade of data linkage in Western Australia: strategic design, applications and benefits of the WA data linkage system. Aust Health Rev 2008; 32: 766-777.

9. Norman PE, Flicker L, Almeida OP, Hankey GJ, Hyde Z, Jamrozik K. Cohort Profile: The Health In Men Study (HIMS). Int J Epidemiol 2009; 38: 48-52.

10. Crum RM, Anthony JC, Bassett SS, Folstein MF. Population-based norms for the Mini-Mental State Examination by age and educational level. JAMA 1993; 269: 2386-2391.

11. McCaul KA, Almeida OP, Norman PE, Yeap BB, Hankey GJ, Golledge J et al. How many older people are frail? Using multiple imputation to investigate frailty in the population. $J$ Am Med Dir Assoc 2015; 16: 439 e431-437.

12. Prince M, Albanese E, Guerchet M, Prina M. World Alzheimer Report 2014 - Dementia and Risk Reduction: an Analysis of Protective and Modifiable Factors. London: Alzheimer's Disease International; 2014.

13. ABS. 3412.0 - Migration, Australia, 2013-14. Canberra: Australian Bureau of Statistics; 2015.

14. Preen DB, Holman CD, Lawrence DM, Baynham NJ, Semmens JB. Hospital chart review provided more accurate comorbidity information than data from a general practitioner survey or an administrative database. J Clin Epidemiol 2004; 57: 1295-1304. 
15. Matthews FE, Stephan BC, Robinson L, Jagger C, Barnes LE, Arthur A et al. A two decade dementia incidence comparison from the Cognitive Function and Ageing Studies I and II. Nat Commun 2016; 7: 11398.

16. Duron E, Funalot B, Brunel N, Coste J, Quinquis L, Viollet C et al. Insulin-like growth factor-I and insulin-like growth factor binding protein-3 in Alzheimer's disease. $J$ Clin Endocrinol Metab 2012; 97: 4673-4681.

17. Hertze J, Nagga K, Minthon L, Hansson O. Changes in cerebrospinal fluid and blood plasma levels of IGF-II and its binding proteins in Alzheimer's disease: an observational study. BMC Neurol 2014; 14: 64.

18. Murialdo G, Barreca A, Nobili F, Rollero A, Timossi G, Gianelli MV et al. Relationships between cortisol, dehydroepiandrosterone sulphate and insulin-like growth factor-I system in dementia. J Endocrinol Invest 2001; 24: 139-146.

19. Tei E, Yamamoto $\mathrm{H}$, Watanabe $\mathrm{T}$, Miyazaki A, Nakadate $\mathrm{T}$, Kato $\mathrm{N}$ et al. Use of serum insulin-like growth factor-I levels to predict psychiatric non-response to donepezil in patients with Alzheimer's disease. Growth Horm IGF Res 2008; 18: 47-54.

20. Vardy ER, Rice PJ, Bowie PC, Holmes JD, Grant PJ, Hooper NM. Increased circulating insulin-like growth factor-1 in late-onset Alzheimer's disease. J Alzheimers Dis 2007; 12: 285290.

21. Johansson P, Aberg D, Johansson JO, Mattsson N, Hansson O, Ahren B et al. Serum but not cerebrospinal fluid levels of insulin-like growth factor-I (IGF-I) and IGF-binding protein-3 (IGFBP-3) are increased in Alzheimer's disease. Psychoneuroendocrinology 2013; 38: 17291737.

22. Dai H, Goto YI, Itoh M. Insulin-like growth factor binding protein-3 deficiency leads to behavior impairment with monoaminergic and synaptic dysfunction. Am J Pathol 2017; 187: 390-400. 


\section{FIGURE LEGEND}

Figure 1. Probability of incident dementia (black line) and respective 95\% confidence interval (grey line) among older men during a mean follow up period of 9 years (range: 0.1 to 13.6) according to their baseline serum concentration of IGF-1 (left panel) and IGFBP-3 (right panel). Probability estimates were derived from logistic regression and the analysis was adjusted for age and history of smoking, diabetes, stroke and body mass index. 


\section{SUPPLEMENTARY MATERIAL}

\section{Systematic review of the association between dementia and IGF-1 and IGFBP-3}

$\underline{\text { Aims }}$

This systematic review aimed to investigate whether the blood concentration (serum or plasma) of IGF-1 and IGFBP-3 was lower among older adults with than without dementia.

\section{$\underline{\text { Study selection }}$}

We used the following search terms on the PubMed database from 1990 to 31 October 2016 to retrieve relevant manuscripts: (insulin-like growth factor OR IGF-1 OR IGF1 OR insulin-like growth factor binding protein OR IGFBP-3 OR IGFBP3) AND (cognitive impairment OR dementia OR Alzheimer). We limited our search to manuscripts published in English. The bibliography of the papers retrieved was examined and additional relevant manuscripts were identified through this process.

We included in the review papers that reported the mean ( \pm standard deviation) serum or plasma concentration of IGF-1 or IGFBP-3 among people with and without dementia. Potentially eligible studies were then rated for quality in five areas: (1) cases and controls were derived from the same population (yes/no/uncertain), (2) the assessment of the exposure was valid (yes/no/uncertain), (3) the diagnosis of dementia was valid (yes/no/uncertain), (4) confounding measures were assessed (yes/no/uncertain), (5) the statistical analyses were appropriate (yes/no/uncertain). Inclusion in the meta-analysis required the relevant study to rate 'yes' for at least items (1), (2) and (3).

\section{$\underline{\text { Statistical analysis }}$}

We managed and analysed the data with the statistical package Stata 14.2 (StataCorp, College Station, Texas 77845, USA). We retrieved the following data from all studies considered acceptable for review: number of participants with and without dementia, mean serum/plasma concentration of 
IGF-1 or IGFBP-3 among participants with and without dementia, standard deviation of IGF-1 or IGFBP-3 among participants with and without dementia. We used the 'metan' command of Stata and the random effects option to analyse the data. Summary data were presented as standardised mean difference (SMD) and respective 95\% confidence interval (95\%CI). Heterogeneity between studies was determined with the I-squared statistic. We used funnel plots to examine the risk of publication bias.

eTable 1

\section{RESULTS}

Five hundred and twenty-four papers were identified using our search strategy, but only 7 reported data potentially suitable for meta-analysis. The quality rating of these studies appears in eTable $1 .^{1-7}$ eTable 2 summarises the data retrieved from the relevant papers. I-squared statistic was $89.9 \%$ for IGF-1 and 87.7\% for IGFBP-3, revealing marked heterogeneity between studies. In addition, funnel plots suggested that publication bias was present (eFigure 1). Of note, 6 of the 7 reports did not take into account the potential impact of confounding measures on their results. ${ }^{2-7}$

eTable 2

eFigure 1

Given the limitations of published studies, we made no attempt to complete a meta-analysis. 


\section{REFERENCES}

1. Duron E, Funalot B, Brunel N, Coste J, Quinquis L, Viollet C et al. Insulin-like growth factor-I and insulin-like growth factor binding protein-3 in Alzheimer's disease. J Clin Endocrinol Metab 2012; 97: 4673-4681.

2. Hertze J, Nagga K, Minthon L, Hansson O. Changes in cerebrospinal fluid and blood plasma levels of IGF-II and its binding proteins in Alzheimer's disease: an observational study. BMC Neurol 2014; 14: 64.

3. Johansson P, Aberg D, Johansson JO, Mattsson N, Hansson O, Ahren B et al. Serum but not cerebrospinal fluid levels of insulin-like growth factor-I (IGF-I) and IGF-binding protein-3 (IGFBP-3) are increased in Alzheimer's disease. Psychoneuroendocrinology 2013; 38: 17291737.

4. Murialdo G, Barreca A, Nobili F, Rollero A, Timossi G, Gianelli MV et al. Relationships between cortisol, dehydroepiandrosterone sulphate and insulin-like growth factor-I system in dementia. J Endocrinol Invest 2001; 24: 139-146.

5. Tei E, Yamamoto H, Watanabe T, Miyazaki A, Nakadate T, Kato $\mathrm{N}$ et al. Use of serum insulin-like growth factor-I levels to predict psychiatric non-response to donepezil in patients with Alzheimer's disease. Growth Horm IGF Res 2008; 18: 47-54.

6. Vardy ER, Rice PJ, Bowie PC, Holmes JD, Grant PJ, Hooper NM. Increased circulating insulin-like growth factor-1 in late-onset Alzheimer's disease. J Alzheimers Dis 2007; 12: 285290.

7. Watanabe T, Miyazaki A, Katagiri T, Yamamoto H, Idei T, Iguchi T. Relationship between serum insulin-like growth factor-1 levels and Alzheimer's disease and vascular dementia. $J$ Am Geriatr Soc 2005; 53: 1748-1753. 

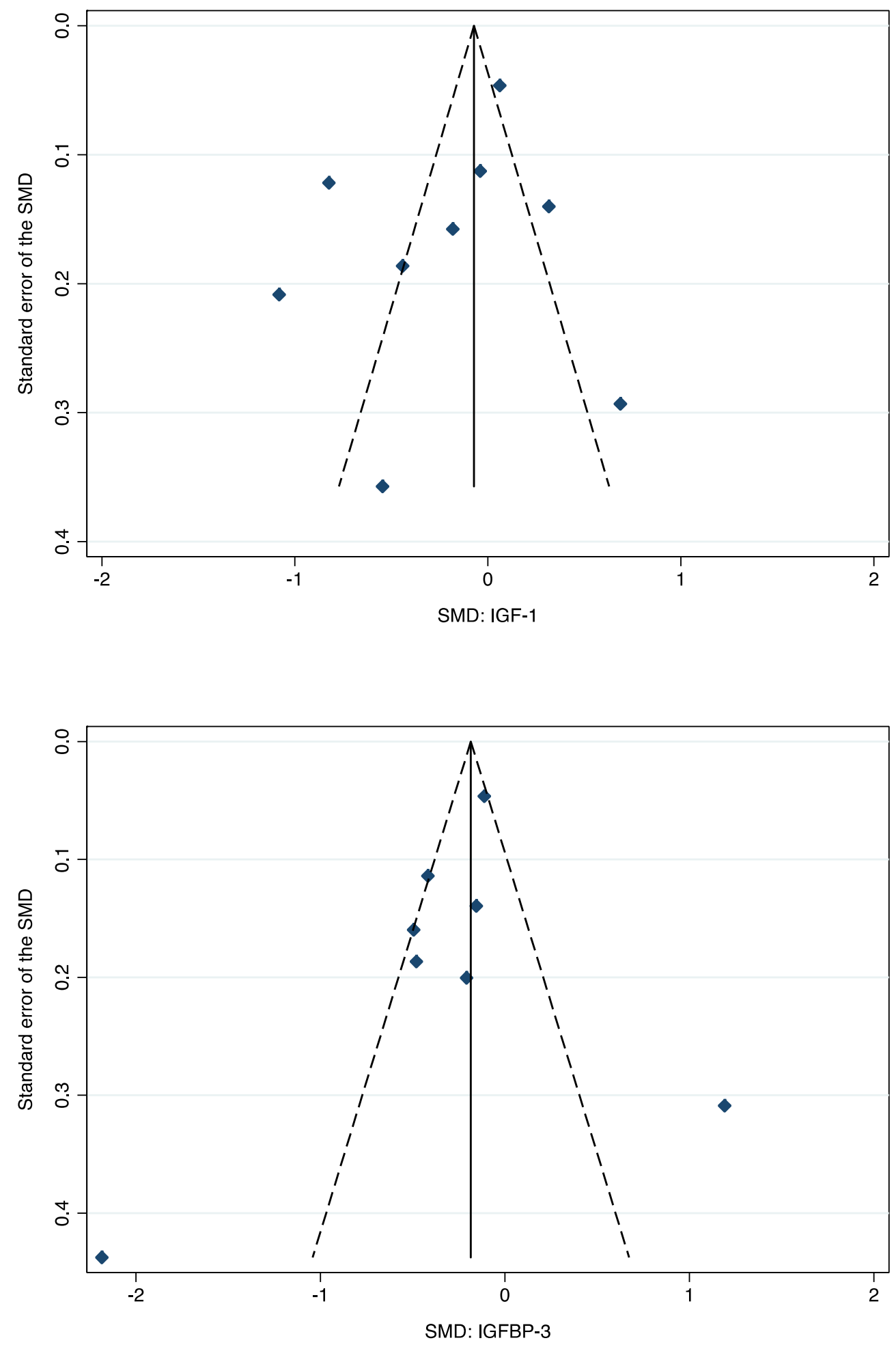

eFigure 1. Funnel plots showing the distribution of studies (diamonds) according to their standardised mean difference (SMD) and the standard error of the SMD for IGF-1 (top graph) and IGFBP-3 (bottom graph). 


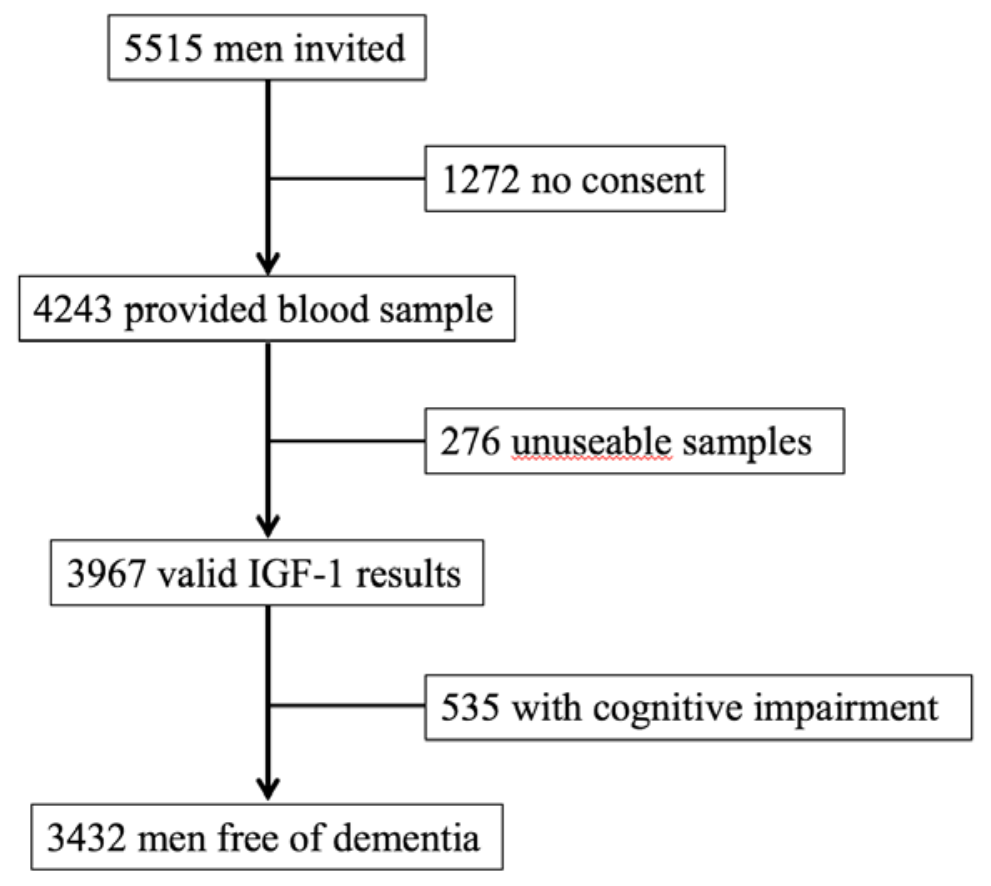

eFigure 2. Flow of participants included in the study: there were 3967 men available for the crosssectional analysis and 3432 for the incident study. 
eTable 2. Summary of the unadjusted results of published studies investigating the association between dementia and IFG-1 and IFGBP-3.

\begin{tabular}{|l|c|c|c|c|c|c|}
\hline Study & \multicolumn{3}{|c|}{ People with dementia } & \multicolumn{3}{c|}{ People without dementia } \\
\hline IGF-1 & N & mean & SD & N & mean & SD \\
\hline Murialdo et al. 2001 (nmol/L) & 25 & 10 & 5.2 & 12 & 14 & 10.7 \\
\hline Watanabe et al. 2005 (ng/mL) & 106 & 119 & 42 & 227 & 160 & 53 \\
\hline Vardy et al. 2007 (nmol/L) & 87 & 11.3 & 2.8 & 126 & 10.3 & 3.4 \\
\hline Tei et al. 2008 (ng/mL) & 50 & 124 & 44.3 & 56 & 173 & 46 \\
\hline Duron et al. 2012 - men $(\mathrm{ng} / \mathrm{mL})$ & 72 & 137 & 69 & 50 & 172 & 92 \\
\hline Duron et al. 2012 - women $(\mathrm{ng} / \mathrm{mL})$ & 152 & 149 & 74 & 163 & 152 & 76 \\
\hline Johansson et al. 2013 (ng/mL) & 32 & 142 & 47.8 & 20 & 110 & 46.6 \\
\hline Hertze et al. 2014 (ng/mL) & 92 & 92 & 39 & 72 & 99 & 38 \\
\hline & & & & & & \\
\hline IGFBP-3 & & & & & & \\
\hline Murialdo et al. 2001 $(\mathrm{nmol} / \mathrm{L})$ & 25 & 49.4 & 12.4 & 12 & 83.9 & 21.4 \\
\hline Vardy et al. 2007 $(\mathrm{nmol} / \mathrm{L})$ & 87 & 65.8 & 19.6 & 126 & 68.7 & 18 \\
\hline Tei et al. 2008 $(\mu \mathrm{gg} / \mathrm{mL})$ & 50 & 2.2 & 0.2 & 56 & 2.2 & 0.3 \\
\hline Duron et al. 2012 $-\mathrm{men}(\mathrm{ng} / \mathrm{mL})$ & 72 & 3675 & 1542 & 50 & 4488 & 1893 \\
\hline Duron et al. 2012 - women $(\mathrm{ng} / \mathrm{mL})$ & 152 & 3781 & 1351 & 163 & 4390 & 1552 \\
\hline Johansson et al. 2013 $(\mu \mathrm{mg} / \mathrm{mL}) *$ & 32 & 3.5 & 0.5 & 20 & 2.9 & 0.5 \\
\hline Hertze et al. 2014 $(\mu \mathrm{g} / \mathrm{mL})$ & 92 & 2 & 0.6 & 72 & 2.3 & 0.6 \\
\hline
\end{tabular}

*Authors reported these results in $\mathrm{ng} / \mathrm{mL}$, but the range of measures suggests that the results are expressed in $\mu \mathrm{g} / \mathrm{mL}$. 
eTable 1. Quality summary of published cross-sectional studies investigating the association between dementia and insulin-like growth factor 1 and insulin-like growth factor binding protein 3.

\begin{tabular}{|c|c|c|c|c|c|c|c|c|c|c|c|c|c|c|c|}
\hline \multirow[t]{2}{*}{ Study } & \multicolumn{3}{|c|}{$\begin{array}{l}\text { Cases and controls from } \\
\text { same population }\end{array}$} & \multicolumn{3}{|c|}{$\begin{array}{l}\text { Valid measurement of } \\
\text { exposure }\end{array}$} & \multicolumn{3}{|c|}{$\begin{array}{c}\text { Valid measurement of } \\
\text { dementia }\end{array}$} & \multicolumn{3}{|c|}{$\begin{array}{l}\text { Valid measurement of } \\
\text { confounding }\end{array}$} & \multicolumn{3}{|c|}{$\begin{array}{l}\text { Appropriate statistical } \\
\text { analyses }\end{array}$} \\
\hline & Yes & No & Unclear & Yes & No & Unclear & Yes & No & Unclear & Yes & No & Unclear & Yes & No & Unclear \\
\hline Murialdo et al. 2001 & & & $\mathrm{X}$ & $\mathrm{X}$ & & & $\mathrm{X}$ & & & & & $\mathrm{X}$ & & & $\mathrm{X}$ \\
\hline Watanabe et al. 2005 & & & $\mathrm{X}$ & $\mathrm{X}$ & & & $\mathrm{X}$ & & & $\mathrm{X}$ & & & & & $\mathrm{X}$ \\
\hline Vardy et al. 2007 & & $\mathrm{X}$ & & $\mathrm{X}$ & & & $\mathrm{X}$ & & & $\mathrm{X}$ & & & & & $\mathrm{X}$ \\
\hline Tei et al. 2007 & & & $\mathrm{X}$ & $\mathrm{X}$ & & & $\mathrm{X}$ & & & $\mathrm{X}$ & & & & & $\mathrm{X}$ \\
\hline Duron et al. 2012 & $\mathrm{X}$ & & & $\mathrm{X}$ & & & $\mathrm{X}$ & & & $\mathrm{X}$ & & & $\mathrm{X}$ & & \\
\hline Johansson et al. 2013 & $\mathrm{X}^{*}$ & & & $\mathrm{X}$ & & & $\mathrm{X}$ & & & $\mathrm{X}$ & & & & & $\mathrm{X}$ \\
\hline Hertze et al. 2014 & & & $X$ & $\mathrm{X}$ & & & $X$ & & & & & $\mathrm{X}$ & & & $\mathrm{X}$ \\
\hline
\end{tabular}

*Cases and controls may have not been independent (e.g., use of control spouses). 
Table 1. Sociodemographic, clinical and biochemical characteristics of participants with and without cognitive impairment at the baseline assessment.

\begin{tabular}{|c|c|c|c|c|c|}
\hline & $\begin{array}{c}\text { No cognitive } \\
\text { impairment } \\
\text { N=3432 } \\
n(\%)\end{array}$ & $\begin{array}{c}\text { Cognitive } \\
\text { impairment } \\
\text { N=535 } \\
\text { n (\%) }\end{array}$ & $\begin{array}{l}X^{2} \text { Statistic } \\
\text { (df) }\end{array}$ & $\mathrm{p}$-value \\
\hline Age (in years) & $\begin{array}{r}70-74 \\
79-79 \\
80-84 \\
\geq 85\end{array}$ & $\begin{array}{c}1325(38.6) \\
1461(42.6) \\
538(15.7) \\
108(3.1)\end{array}$ & $\begin{array}{c}164(0.6) \\
229(42.8) \\
106(19.8) \\
36(6.7)\end{array}$ & $29.05(3)$ & $<0.001$ \\
\hline \multicolumn{2}{|c|}{ Education: completed high school } & $1736(50.6)$ & $183(34.3)$ & $49.33(1)$ & $<0.001$ \\
\hline Smoking history & $\begin{array}{r}\text { Never } \\
\text { Past } \\
\text { Current }\end{array}$ & $\begin{array}{c}1168(34.0) \\
2088(60.8) \\
176(5.1)\end{array}$ & $\begin{array}{c}171(32.0) \\
334(62.4) \\
30(5.6)\end{array}$ & $0.98(2)$ & 0.611 \\
\hline \multicolumn{2}{|l|}{ Diabetes } & 475 (13.8) & 74 (13.8) & $0.00(1)$ & 0.996 \\
\hline \multicolumn{2}{|l|}{ Hypertension } & $1617(47.1)$ & $268(50.1)$ & $1.65(1)$ & 0.199 \\
\hline \multicolumn{2}{|c|}{ Coronary heart disease } & $991(28.9)$ & $181(33.8)$ & $5.46(1)$ & 0.019 \\
\hline \multicolumn{2}{|l|}{ Stroke } & $419(12.2)$ & 98 (18.3) & $15.24(1)$ & $<0.001$ \\
\hline & & Mean \pm SD & Mean \pm SD & t-test $(\mathrm{df})^{\#}$ & p-value ${ }^{\#}$ \\
\hline \multicolumn{2}{|c|}{ Body mass index $\left(\mathrm{Kg} / \mathrm{m}^{2}\right)$} & $26.6 \pm 3.6$ & $26.5 \pm 3.7$ & $0.51(3952)$ & 0.611 \\
\hline \multicolumn{2}{|l|}{ IGF-1 (ng/ml) } & $140.8 \pm 57.9$ & $144.4 \pm 64.5$ & $0.72(3956)$ & 0.469 \\
\hline \multicolumn{2}{|l|}{ IGFBP-3 (ng/ml) } & $3799.2 \pm 903.2$ & $3697 \pm 911.0$ & 2.40 (3965) & $0.016 *$ \\
\hline
\end{tabular}

"Statistical tests based on the natural logs of IGF-1 and IGFBP-3.

${ }^{*} \mathrm{p}=0.292$ after the analyses were adjusted for age (in years), educational attainment and past history of coronary heart disease or stroke. (Educational attainment was included in these analyses because it was associated with both cognitive impairment and IGFBP-3.) 
Table 2. Crude and adjusted hazard ratio (HR) of incident dementia associated with IGF-1 and IGFBP-3 measures over an average follow up period of 9 years.

\begin{tabular}{|l|c|c|}
\hline & Crude HR $(95 \% \mathrm{CI})$ & Adjusted HR $(95 \% \mathrm{CI})^{*}$ \\
\hline IGF-1: doubling & $1.03(0.90,1.18)$ & $1.04(0.91,1.18)$ \\
\hline IGF-1: $1^{\text {st }}$ quintile & 1 (reference) & 1 (reference) \\
\hline IGF-1: $2^{\text {nd }}$ quintile & $1.10(0.86,1.42)$ & $1.12(0.87,1.45)$ \\
\hline IGF-1: $3^{\text {rd }}$ quintile & $1.18(0.91,1.52)$ & $1.18(0.92,1.53)$ \\
\hline IGF-1: $4^{\text {th }}$ quintile & $0.90(0.69,1.18)$ & $0.91(0.70,1.20)$ \\
\hline IGF-1: $5^{\text {th }}$ quintile & $1.12(0.86,1.46)$ & $1.13(0.86,1.47)$ \\
\hline & & $0.76(0.62,0.94)$ \\
\hline IGFBP-3: doubling & $0.73(0.59,0.89)$ & $1($ reference) \\
\hline IGFBP-3: $1^{\text {st }}$ quintile & $1($ reference $)$ & $0.80(0.62,1.02)$ \\
\hline IGFBP-3: $2^{\text {nd }}$ quintile & $0.78(0.61,0.99)$ & $0.87(0.68,1.11)$ \\
\hline IGFBP-3: $3^{\text {rd }}$ quintile & $0.84(0.66,1.07)$ & $0.71(0.54,0.92)$ \\
\hline IGFBP-3: $4^{\text {th }}$ quintile & $0.68(0.52,0.88)$ & $0.71(0.54,0.93)$ \\
\hline IGFBP-3: $5^{\text {th }}$ quintile & $0.68(0.52,0.89)$ & \\
\hline
\end{tabular}

95\%CI: 95\% confidence interval of the hazard ratio.

*Analyses adjusted for age, smoking group, and history of diabetes, coronary heart disease, stroke and body mass index. (Education was not included in the adjusted analyses because it did not affect the risk estimate of dementia; $\mathrm{p}=0.349$ ) 